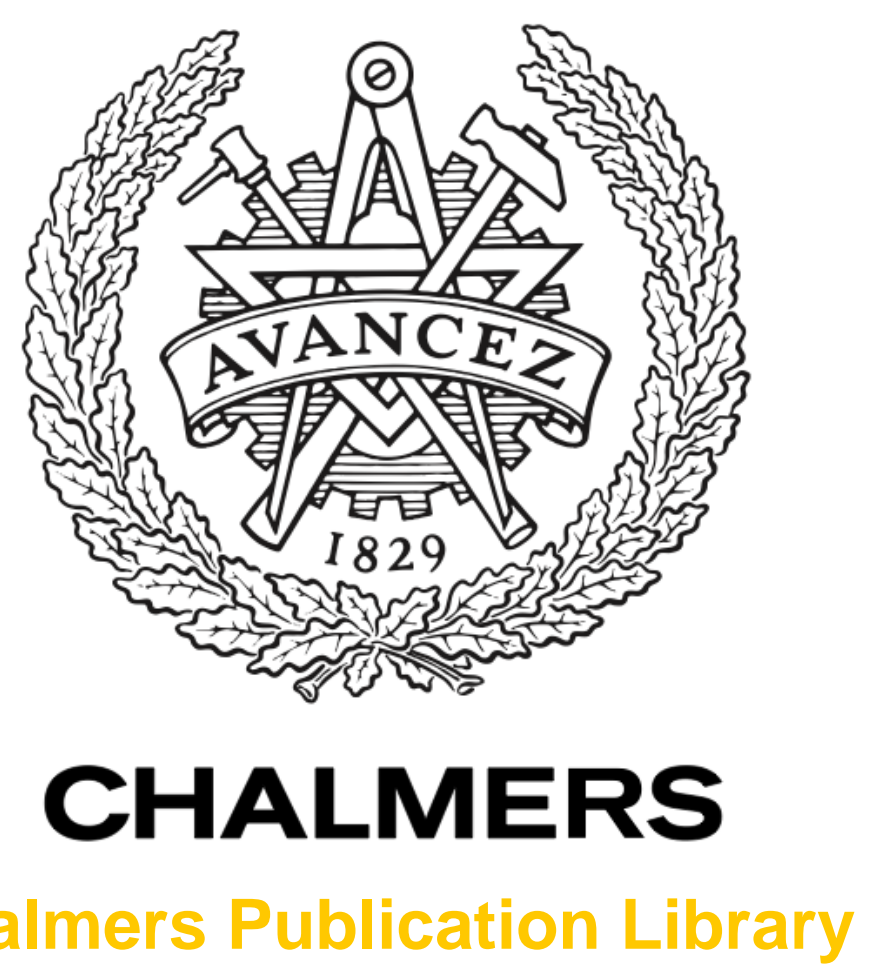

\title{
Modelling plasma response to RMP fields in ASDEX Upgrade with varying edge safety factor and triangularity
}

This document has been downloaded from Chalmers Publication Library (CPL). It is the author's version of a work that was accepted for publication in:

Nuclear Fusion (ISSN: 00295515)

Citation for the published paper:

Li, L. ; Liu, Y. ; Kirk, A. et al. (2016) "Modelling plasma response to RMP fields in ASDEX Upgrade with varying edge safety factor and triangularity". Nuclear Fusion, vol. 56(12), pp. Art. no. 126007.

http://dx.doi.org/10.1088/0029-5515/56/12/126007

Downloaded from: http://publications.lib.chalmers.se/publication/247840

Notice: Changes introduced as a result of publishing processes such as copy-editing and formatting may not be reflected in this document. For a definitive version of this work, please refer to the published source. Please note that access to the published version might require a subscription. 


\section{Modelling plasma response to RMP fields in ASDEX-Upgrade with varying edge safety factor and triangularity}

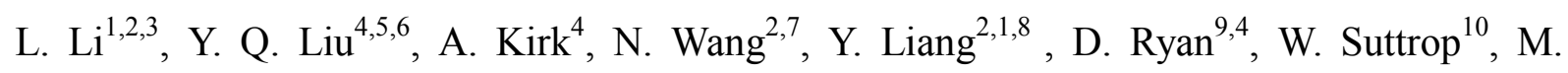
Dunne $^{10}$, R. Fischer ${ }^{10}$, J.C. Fuchs ${ }^{10}$, B. Kurzan ${ }^{10}$, M. Willensdorfer ${ }^{10}$, F. C. Zhong ${ }^{1,3}$ and the ASDEX Upgrade team ${ }^{10}$ and the EUROfusion MST1 team ${ }^{11}$

${ }^{1}$ College of Science, Donghua University, Shanghai 201620, China

${ }^{2}$ Forschungszentrum Jülich GmbH, Institut für Energie- und Klimaforschung - Plasmaphysik, Jülich, Germany

${ }^{3}$ Member of Magnetic Confinement Fusion Research Centre, Ministry of Education, China

${ }^{4}$ CCFE, Culham Science Centre, Abingdon, OX14 3DB, UK

${ }^{5}$ Southwestern Institute of Physics, PO Box 432, Chengdu 610041, China

${ }^{6}$ Department of Earth and Space Science, Chalmers University of Technology, SE-412 96 Gothenburg, Sweden

${ }^{7}$ State Key Laboratory of Advanced Electromagnetic Engineering and Technology, Huazhong University of Science and

Technology, Wuhan, Hubei 430074, China

${ }^{8}$ Institute of Plasma Physics, Chinese Academy of Sciences, P.O. Box 1126, Hefei230031, China

${ }^{9}$ Department of Physics, University of York, Heslington, York, YO10 5DD, UK

${ }^{10}$ Max Planck Institute for Plasma Physics, Boltzmannstr. 2, 85748 Garching, Germany

${ }^{11}$ See http://www.euro-fusionscipub.org/mst1

"E-Mail: 1.li@fz-juelich.de

\section{Abstract}

Toroidal computations are performed using the MARS-F code [Liu Y Q et al 2000 Phys.

Plasmas 7 3681], in order to understand correlations between the plasma response and the observed mitigation of the edge localized modes (ELM) using resonant magnetic perturbation fields in ASDEX Upgrade. In particular, systematic numerical scans of the edge safety factor reveal that the amplitude of the resonant poloidal harmonic of the response radial magnetic field near the plasma edge, as well as the plasma radial displacement near the X-point, can serve as good indicators for predicting the optimal toroidal phasing between the upper and lower rows of coils in ASDEX Upgrade. The optimal coil phasing scales roughly linearly with the edge safety factor $q_{95}$, for various choices of the toroidal mode number $n=1-4$ of the coil configuration. The optimal coil phasing is also predicted to vary with the upper triangularity of the plasma shape in ASDEX Upgrade. Furthermore, multiple resonance 
effects of the plasma response, with continuously varying $q_{95}$, are computationally observed and investigated.

\section{Introduction}

Edge localized modes (ELMs) are common MHD instabilities observed in many present tokamaks operating in the so called H-mode. ELMs manifest themselves with periodic bursts near the plasma edge, accompanied by large particle and heat fluxes leaving the plasma and reaching the facing material components. The material damage, caused by the so called type-I ELMs, can be potentially dangerous for future fusion devices such as ITER [1]. Therefore, suppression or mitigation of the type-I ELMs presents a crucial challenge as long as a fusion reactor is to be operated in $\mathrm{H}$-mode.

Among various techniques proposed to control type-I ELMs, the resonant magnetic perturbation (RMP), provided by magnetic coils surrounding the torus, has been shown to be often effective, in many present day tokamak devices [2-7]. Despite significant efforts during recent years [8-19], theoretical understanding of the ELM control physics with RMP fields is so far incomplete. It is, however, well established now that the plasma response plays a significant role in the ELM mitigation, possibly also in the ELM suppression. Substantial efforts have thus been devoted in computational modelling of the plasma response to RMP fields, in realistic toroidal geometry $[8,10-12,15-18]$.

ASDEX Upgrade is presently equipped with two rows of ELM control coils, one located near the top, and the other near the bottom, of the plasma surface, with both rows being on the low field side. Experiments have demonstrated that the toroidal phase difference (the so called coil phasing) between the coil currents of the upper and lower rows, is one of 
the key factors affecting the ELM mitigation in ASDEX Upgrade [20]. More interestingly, the optimal coil phasing appears to be varying with plasma parameters. Previous modelling work has pointed to a correlation between the ELM mitigation and edge peeling response in both MAST [11] and ASDEX Upgrade [17, 20] plasmas. The peeling mode response, in turn, is in good correlation with the peaking of the plasma displacement near the X-point [11].

Besides the X-point displacement, caused by the plasma response to the applied RMP fields, we shall also consider another figure of merit, namely the amplitude of the pitch resonant poloidal harmonic of the computed perturbed radial magnetic field near the plasma edge. It has been found before $[11,20]$, and will be extensively demonstrated in this work, that both of these figures of merit result in the same prediction for the optimal coil phasing, for the ASDEX Upgrade plasmas considered in this work.

We shall computationally examine the aforementioned figures of merit, with systematic scans of $q_{95}$ and plasma shaping, in order to determine the optimal coil phasing. ASDEX Upgrade, like many other tokamak devices, has the flexibility of operating at different safety factor values, by either changing the plasma current or the toroidal vacuum field. So far the ELM control experiments in different tokamak devices have been carried out with different plasma shapes, which may or may not be similar to the shape of the ITER target plasmas. Joint experimental work, between ASDEX Upgrade and DIII-D has recently been initiated, in order to investigate the effect of the plasma shaping on the ELM control. However, a systematic theory study on this issue is still absent. This partly motivates our work presented here. In particular, since ASDEX Upgrade has more flexibility in modifying the upper triangularity of the plasma separatrix (the lower triangularity is more constrained by the location of the lower divertor strike point), we shall study the sensitivity of the plasma response with respect to the variation of the upper triangularity, based on an ASDEX Upgrade 
equilibrium. The modelling results thus can be useful in guiding the planned experiments on ASDEX Upgrade in the near future, where both the plasma shaping and the $q_{95}$ effects will be investigated. In particular, our modelling results provide guidance on the choice of the optimal coil phasing in these ELM control experiments with various plasma configurations.

The modelling is carried out using the MARS-F code [21], which has been well benchmarked $[22,23]$ and extensively applied to model the plasma response to external 3D fields. With eight evenly spaced RMP coils in ASDEX Upgrade, the possible choices of the toroidal mode number are $n=1,2,4$. All three coil configurations are thus investigated in this work. With the limited number of the coil power supplies (presently 4 in total), the $n=3$ field can also be produced, but with significant sidebands. In the modelling, we shall also consider the plasma response to the $n=3$ field, but neglecting the sideband effects.

The next Section introduces the plasma and coil configurations in ASDEX Upgrade experiments, as well as a brief description of the MARS-F computational model. Section 3 reports the $q_{95}$ scan results. Comparison is also made between the modelling and experiments. Section 4 reports computational results for the optimal coil phasing, when the upper triangularity of the ASDEX Upgrade plasma shape is gradually modified. Section 5 summarizes the work.

\section{Plasma-coil configuration and MARS-F formulation}

\subsection{Plasma equilibrium}

An ASDEX Upgrade equilibrium has been reconstructed, based on discharge 30835 at 3200 ms. Shown in Fig. 1(a) is the plasma boundary shape for this equilibrium. For the reason of numerical accuracy, the original plasma shape (black line) is slightly modified near the $\mathrm{X}$ - 
point (blue line). This modification decreases the value of the safety factor at the plasma surface, without appreciable change of other equilibrium parameters, including the total plasma current and the safety factor $q$ at $95 \%$ of magnetic flux surface $\left(q_{95}\right)$. The plasma response is also not significantly affected by such a smoothing procedure [17].

Discharge 30835 has a low safety factor, with $q_{95}=3.719$. The equilibrium radial profiles at $3200 \mathrm{~ms}$ were reported in [17]. In order to investigate the effect of $q_{95}$ on the plasma response to the RMP fields, as well as the dependence of the optimal coil phasing on $q_{95}$, we consider a series of numerical equilibria by gradually varying the total plasma current $I_{p}$ (at fixed toroidal equilibrium field). This results in $q_{95}$ varying between 3.1 and 6.1 . Examples of the computed $q$-profiles are shown in Fig. 1(b).

\subsection{RMP coils configuration}

The two sets of ELM control coils (B-coils) in ASDEX Upgrade are located at the low field side of the torus, as shown in Fig. 1(a). Since there are 8 coils in each row, magnetic field perturbations, with toroidal mode number $n=1,2$ and 4, can be produced. In experiments, the toroidal phase of the applied magnetic field can be tuned by varying currents in the upper and lower rows of coils. For the $n=1$ and $n=2$ configurations, 8 coils are sufficient to yield continuous variation of the toroidal phasing between the upper and lower rows. For the $n=4$ configuration, however, there are only two possible choices of the coil phasing, i.e. either even parity or odd parity. On the other hand, the MARS-F modelling allows continuous variation of the coil phasing for all n's, by assuming larger number of coils per row.

\subsection{MARS-F computational model for the plasma response}

In this work, we use the MARS-F code [21] to compute the toroidal plasma response to external 3D magnetic fields produced by B-coils in ASDEX Upgrade. The code solves 
linearized single fluid, resistive, full MHD equations in toroidal geometry, in the presence of toroidal flow as measured in experiments. Besides the plasma region described by MHD equations, the MARS-F model also includes a real vacuum region, as well as current carrying coils in the vacuum.
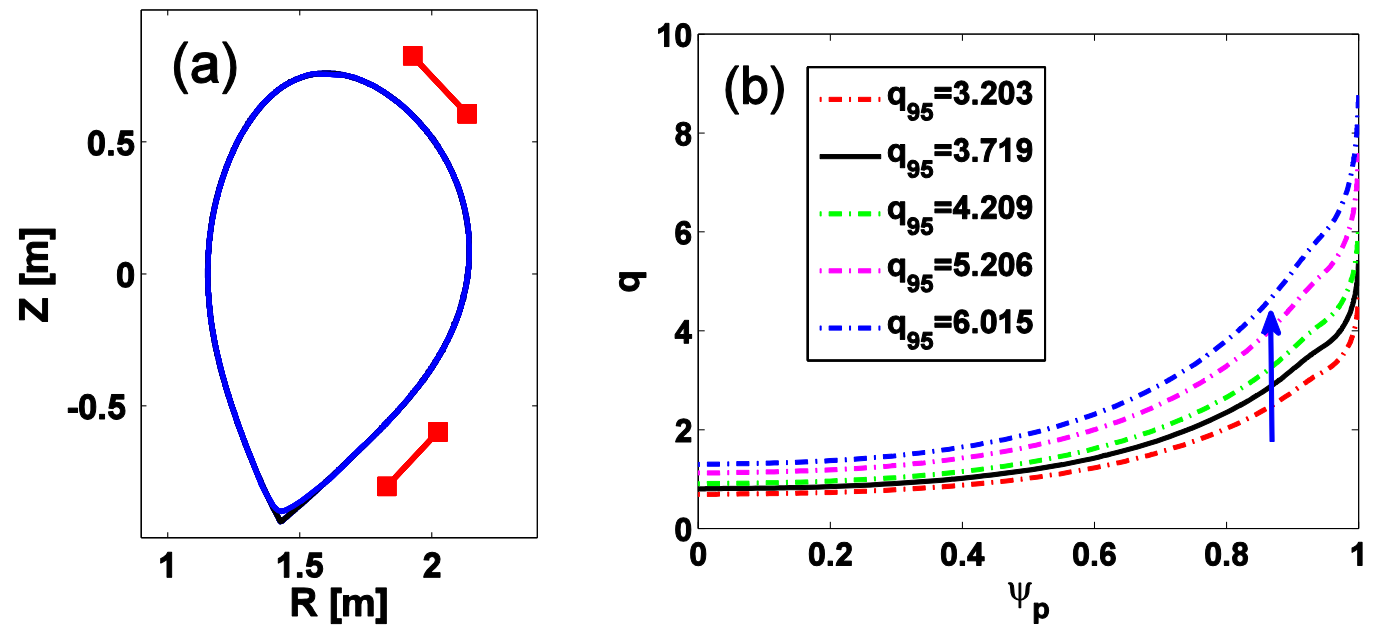

Figure 1. (a) The poloidal cross section of the plasma boundary shape (black line), slightly smoothed near the Xpoint (blue line), for ASDEX Upgrade discharge 30835 at $3200 \mathrm{~ms}$. The upper and lower rows of ELM control coils are shown by red lines. The corresponding radial profile of the safety factor $q$ is shown by the black curve in (b), together with a series of numerically assumed q-profiles with varying $q_{95}$ (by varying the total plasma current). $\psi_{p}$ is the normalized equilibrium poloidal magnetic flux labeling the plasma minor radius.

For plasma response computations, the RMP coil currents are taken into consideration as the source terms in the code. For a given toroidal phase difference between the upper and lower sets of coil currents, $\Delta \Phi=\Phi^{\text {upper }}-\Phi^{\text {lower }}$, and the coil current amplitude, we can directly compute the plasma response to the combined fields by two rows of coils. As an example, Fig. 2(a) compares the amplitude of the pitch aligned resonant radial field components, at the corresponding rational surface locations, between the vacuum field and the total field including the MARS-F computed plasma response. The original equilibrium for 30835 with $q_{95}=3.719$, is considered here. The B-coils in the $n=2$ configuration and in even 
parity are assumed. The radial field component, in a generic toroidal flux coordinate system, is defined here as

$$
b^{1}=\left(\frac{\mathbf{b} \cdot \nabla \psi}{\mathbf{B}_{\mathrm{eq}} \cdot \nabla \phi}\right) \frac{q}{R_{0}^{2} B_{0}},
$$

where $\mathbf{b}$ is perturbed magnetic field, $\psi$ the equilibrium poloidal magnetic flux, $\mathbf{B}_{e q}$ the equilibrium field, $R_{0}$ the major radius of the torus, $B_{0}$ the on-axis vacuum toroidal field strength. Note that $b^{1}$ is a dimensionless quantity. Normally the resistive plasma response, in the presence of toroidal flow, substantially screens the resonant components of the vacuum field, as has been previously reported [8, 24]. Less screening, however, occurs near the plasma edge, where the plasma resistivity is large (following the Spitzer scaling of $T_{e}^{-3 / 2}$ ) and the toroidal flow is relatively slow (compared to the core flow).

In the following, we shall define two figures of merit (vacuum versus plasma response) based on the amplitude of the outermost resonant poloidal harmonic, while scanning $q_{95}$ and the coil phasing parameter $\Delta \Phi$. More specifically, we define $b^{l}{ }_{r e s}=b^{1}{ }_{m n}(q=m / n)$, where $b^{1}$ is defined in the above Eq. (1), and $m$ is the largest poloidal harmonic number within the plasma surface (with finite $q_{a}$ value). It is well known that the amplitude of the resonant radial field perturbation is proportional to the square of the magnetic island width.

The other figure of merit, that we define, is based on the normal displacement of the plasma surface, as a result of the plasma response to the RMP fields. Figure 2(b) shows the MARS-F computed amplitude of the normal displacement along the poloidal angle, for the 30835 plasma. In particular, we are interested in the plasma displacement near the X-point, defined as the local peak amplitude near $\theta=-107^{\circ}$ for this ASDEX Upgrade equilibrium. 
The choices of aforementioned two figures of merit are motivated by the characterization of the plasma response to external 3D fields. It has been found that there are essentially two kinds of plasma response to the external magnetic field perturbations, produced by the RMP coils [11]. One is the core kink response, which has a global structure in terms of the plasma radial displacement, and is thought to be triggered by the response of a stable core kink mode to the applied field. The kink response can often be substantially reduced by strong parallel sound wave damping [19]. The other type of the plasma response is due to the edge localized, stable peeling mode. This response, which is relatively insensitive to the sound wave damping, is well described by the amplitude of the resonant radial field harmonics near the plasma edge, as well as the plasma surface displacement near the X-point [17]. More interestingly, previous work has established a good correlation between the computed peeling type of the plasma response on one side, and on the other side the observed RMP effects on the plasma density pump out and on the type-I ELM mitigation in MAST [11, 25] and ASDEX Upgrade [20] experiments.
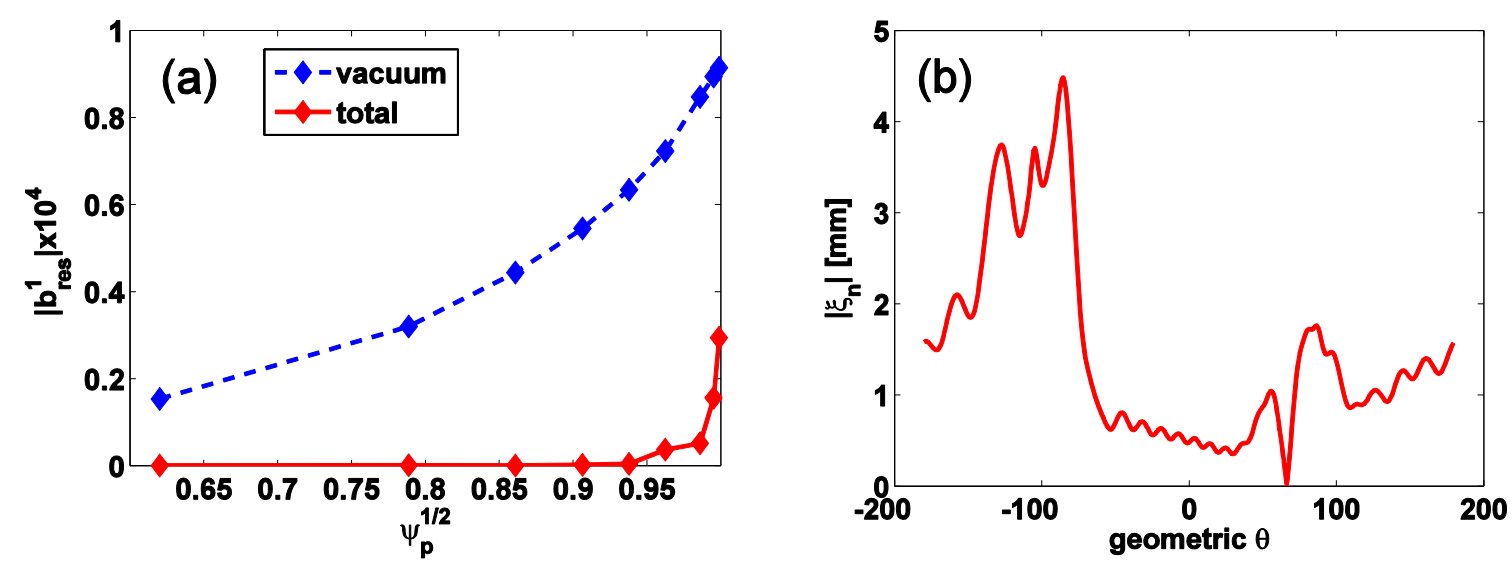

Figure 2. The MARS-F computed (a) amplitude of the pitch resonant radial magnetic field components at the corresponding rational surface locations, for the vacuum RMP field (blue dashed line) and the total field (red solid line) including the plasma response, and (b) amplitude of the normal displacement of the plasma surface along the geometric poloidal angle, where $\theta=0^{\circ}$ corresponds to the outboard mid-plane, and $\theta=-107^{\circ}$ corresponds to the location of the X-point. Chosen is the 30835 equilibrium at $3200 \mathrm{~ms}$, with $q_{95}=3.719$. The RMP coils are assumed to be in even parity $\left(\Delta \Phi=0^{\circ}\right), n=2$ configuration. 


\section{Effects of $q_{95}$ on the plasma response and the optimal coil phasing}

Experiments in JET [26] and ASDEX Upgrade [20] have demonstrated that the ELM mitigation capability of a given coil configuration is sensitive to the variation of $q_{95}$. Computational results of dense scan of $q_{95}$ are reported below, for different choices of the toroidal mode number for the applied RMP field perturbation. Since majority of the ELM control experiments in ASDEX Upgrade have so far been carried out with the $n=2$ coil configuration, we report the $n=2$ results first.

\subsection{Plasma response to $n=2$ RMP field}

Figure 3 summarizes the MARS-F computated three figures of merits as defined in Section 2.3, for a series of equilibria with $q_{95}$ varying between 3.1 and 6.1. At each $q_{95}$, the coil phasing angle $\Delta \Phi$ varies between $-180^{\circ}$ and $180^{\circ}$. The $2 \mathrm{D}$ plots in Fig. 3(a-b) show the amplitude of the last resonant vacuum radial field. Plots in Fig. 3(c-d) show the amplitude of the last resonant radial field component with the plasma response. Plots in Fig. 3(e-f) show the amplitude of the X-point displacement. In order to clearly show the optimal coil phasing, the figures of merit on the left panel plots $(\mathrm{a}, \mathrm{c}, \mathrm{e})$ are linearly scaled to the $[0,1]$ range, at each $q_{95}$. Experimental data points of the ELM mitigation (mitigated versus non-mitigated cases in the $\left(q_{95}, \Delta \Phi\right)$ space $)$ are also shown in the left panel plots.

Several interesting observations can be made from Fig. 3. First, the left panel shows that the optimal coil phasing, that maximizes the figures of merit, scales roughly linearly with $q_{95}$, as indicated by solid straight lines in plots (a, c, e). In other words, the relation between the optimal phasing angle $\Delta \Phi$ and $q_{95}$ is well approximated by the following formula 
$n q_{95}=a_{n}\left(\Delta \phi+180^{\circ}(2 k+1)+\phi_{n}\right)$

where $k$ are integers. The slope coefficient $a_{n}$ and the phase shift factor $\phi_{n}$ can be well determined, based on each of the figure of merit. We find that, for the $n=2$ coil configuration, $a_{n}=0.026$ for all three figures of merit. However, $\phi_{n}=-50^{\circ}$ according to the vacuum radial field, whilst $\phi_{n}=10^{\circ}$ according to both the plasma response radial field and the $\mathrm{X}$-point displacement criteria. The plasma response generally introduces a $60^{\circ}$ phase shift for the optimal coil phasing, as compared to the vacuum field. This $60^{\circ}$ phase shift was also computationally observed in previous work [17], but only for isolated $q_{95}$ values. The plasma response, on the other hand, does not change the slope coefficient $a_{n}$. It is important to point out that both plasma response based figures of merit (c, e) yield the same phase shift factor $\phi_{n}$ and thus the same optimal choice for the coil phasing.

The "worst" coil phasing, that minimizes the figures of merit, also follows straight lines in the 2D space $\left(q_{95}, \Delta \Phi\right)$, as shown by the dashed lines in the left panel plots (a, c, e). In fact, the worst phase is always $180^{\circ}$ apart from the optimal coil phasing, and the analytic approximation reads

$$
n q_{95}=a_{n}\left(\Delta \phi+180^{\circ}(2 k+0)+\phi_{n}\right)
$$

where the slope coefficient $a_{n}$ and the phase shift factor $\phi_{n}$ are the same as for the optimal coil phasing, obtained with the corresponding figure of merit. 

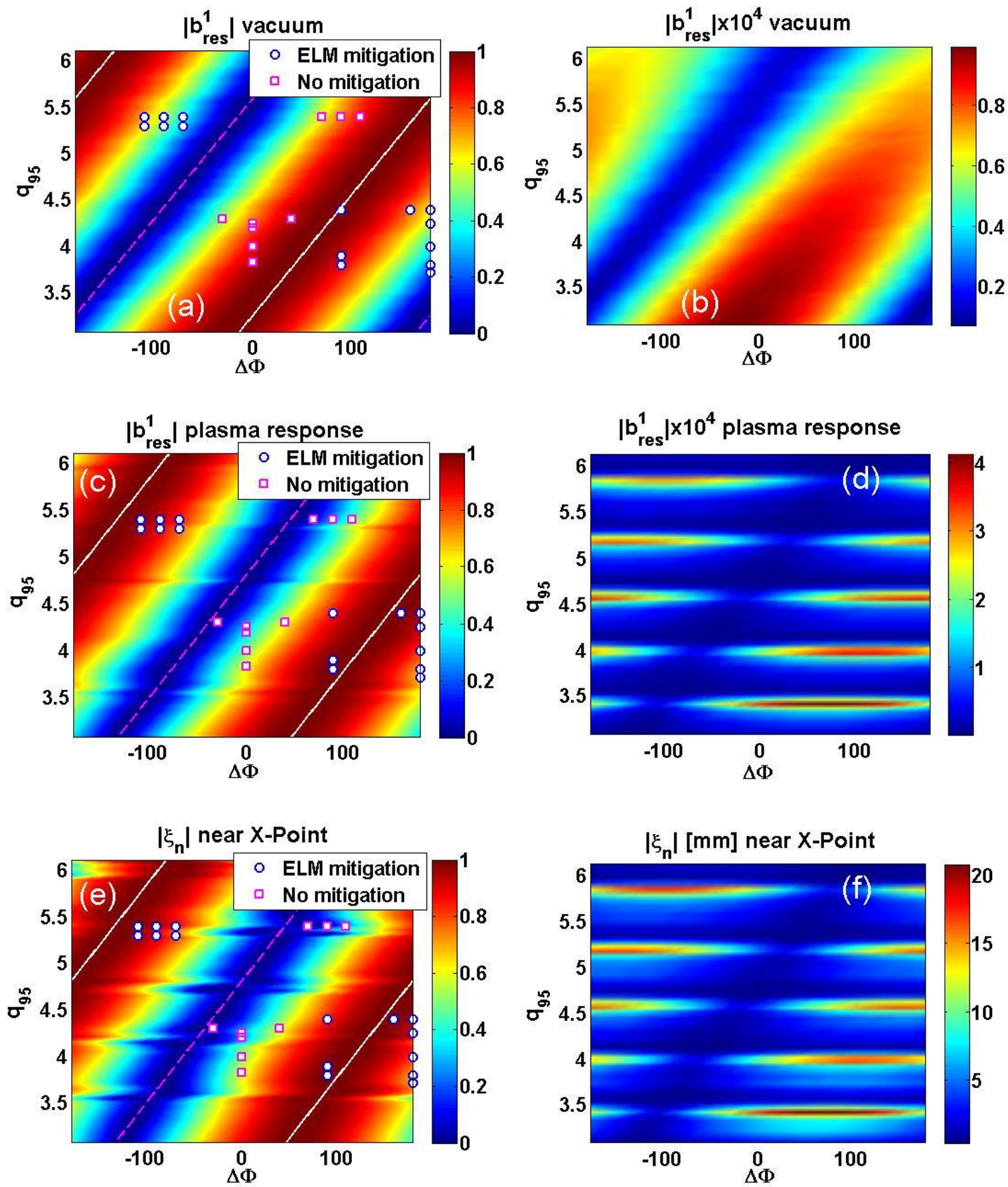

Figure 3. Various figures of merit computed in $2 \mathrm{D}$ parameter space $\left(\Delta \Phi, q_{95}\right)$, for (a-b) the amplitude of the outermost pitch resonant vacuum radial field component; (c-d) the amplitude of the outermost pitch resonant radial field component including the plasma response; and (e-f) the amplitude of the plasma surface displacement near the X-point. These quantities are all re-scaled to the range of $[0,1]$, at each given $q_{95}$, in the left panel plots (a, c, e), where the solid lines indicate the location of the peak value (of 1), and the dashed lines indicate the location of the minimum (of 0). Experimental data points, with ELM mitigation being achieved (open circles) and not being achieved (open squares), are also added into the left panel. Here $\Delta \Phi$ is defined as 
the toroidal phase difference (in degrees) for the coil currents between the upper and the lower rows. The $n=2$ coil configuration is considered.

The second, and probably the most significant, observation from Fig. 3(a, c, e), is that the plasma response based figures of merit (c, e) generally correctly recover the best coil phasing for the ELM mitigation as achieved in experiments. The ELM mitigation here refers to those discharges (shot numbers 31143, 31144, 31945, 32080, 32085, 32086, 32089, 32091, 32092), where frequency of the type-I ELMs is increased in experiments, after applying the RMP fields. In the no-mitigation discharges (shot numbers 31944, 32081, 32320, 32321), no increase of the ELM frequency has been observed. The plasma-coil configurations in the $\left(q_{95}, \Delta \Phi\right)$ space, where no ELM mitigation was achieved in experiments, align well with the coil phasing that tends to minimize the plasma response based figures of merit. On the other hand, the vacuum field figure of merit (a) does not give a consistent prediction for the ELM mitigation in experiments. The ELM control experiments in ASDEX Upgrade have so far been exploiting limited regions of the $q_{95}$ space. The analytic formula found here can thus be used to guide the choice of the coil phasing for a wide variation of $q_{95}$.

We now discuss the third interesting computational observation, shown by the right panel of Fig. 3. The same figures of merit are shown here, but without re-normalization at each $q_{95}$. The last resonant component of the vacuum field gradually decreases with increasing $q_{95}$, as shown by plot (b). This is understandable, since the amplitude of the vacuum field component $m \sim n q_{95}$ generally decreases with the poloidal mode number $m$. The computed plasma response, however, experiences strong amplification at multiple values of $q_{95}$. This multiple resonance effect, also previously found in DIII-D modelling [18], has been shown to be related to the stability property of the edge localized peeling mode [27]. 

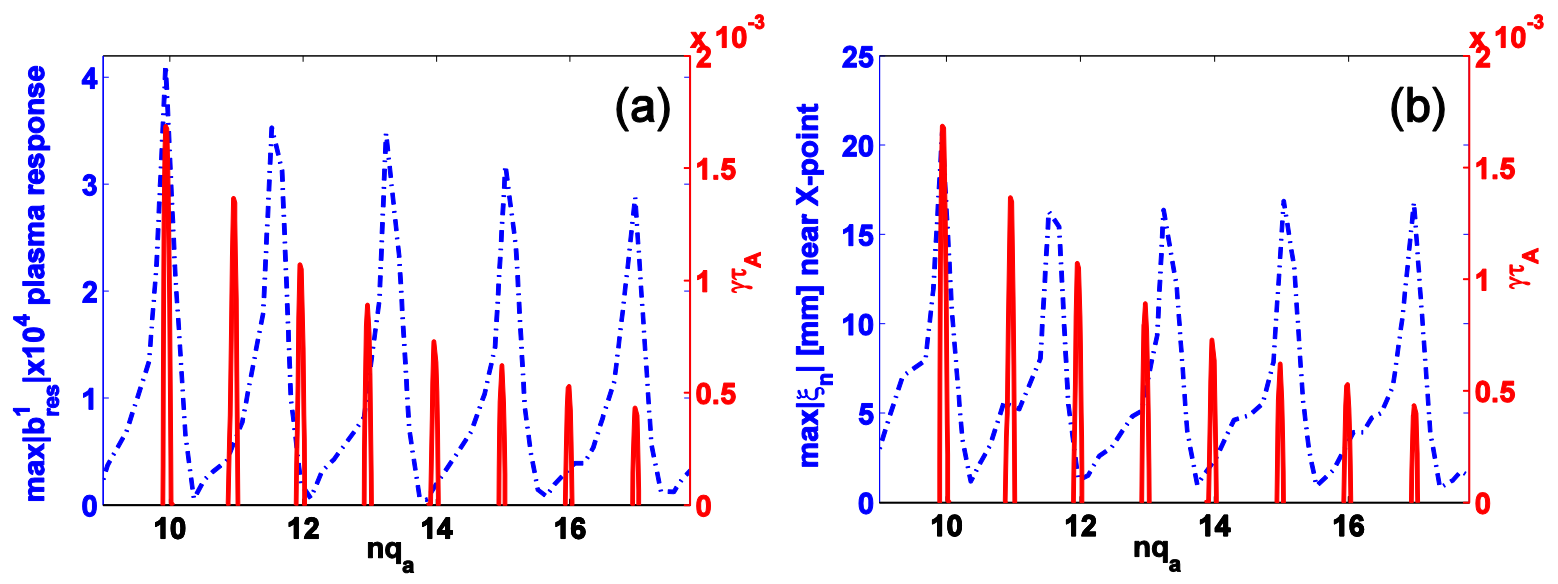

Figure 4. Growth rates (solid curves with right vertical axis in both plots, normalized by the on-axis toroidal Alfvén time) of the computed $n=2$ edge localized ideal peeling instability versus the edge safety factor $q_{a}$, plotted together with (a) the maximal amplitude (over all coil phasing angles $\Delta \Phi$ at fixed safety factor) of the last pitch resonant radial magnetic field component including the plasma response shown by the dash-dotted curve, and (b) the maximal amplitude of the X-point displacement shown by the dash-dotted curve, for the $n=2$ ELM control coil configuration.

In order to more clearly illustrate this correlation, Fig. 4(a) and (b) plots the peak value (over the coil phasing at each $q_{95}$ ) of the two plasma response based figures of merit (dashdotted curves), respectively, versus the edge safety factor $q_{a}$ multiplied by the toroidal mode number $n(=2)$. We choose $q_{a}$ instead of $q_{95}$ as the dependent variable here, since the ideal peeling mode stability, shown by the solid curves with the right hand side vertical axis, is directly associated with the $q_{a}$ value - the mode's growth rate crosses marginal stability point at integer numbers for $n q_{a}$. In our numerical study, the plasma boundary surface has been slightly smoothed near the X-point (see Fig. 1(a)), leading to finite edge $q$-values. Figure 4 shows that there is no one-to-one correspondence between the plasma response peaking and the ideal peeling mode stability, but clearly there are several cases, where the large response due to the plasma amplification occurs in the stable window for the ideal peeling mode. We note that, in fact, within linear theory assumption, only the response computed in the stable window has physics significance, although MARS-F, owing to its special numerical algorithm, allows us to compute the stationary response point of an even unstable linear system. More 
discussions will be made, on the correlation between the multi-resonance in the plasma response and the peeling mode stability, in the following subsection, where the computational results for the $n=1$ RMP coil configuration are reported.

\subsection{Plasma response to $n=1$ RMP field}

Limited number of ELM control experiments have recently been performed in ASDEX Upgrade using the $n=1$ coil configuration, due to high probability of causing the $n=1$ mode locking. Clear ELM mitigation was observed though in early campaigns, using the $n=1$ RMP fields [28]. For the purpose of modelling the $n=1$ experiments, as well as for the comparison with the $n=2$ results, here we choose the same plasma equilibria based on discharge 30835 at $3200 \mathrm{~ms}$, as for the $n=2$ coil configuration presented in the previous subsection, and perform the systematic plasma response scan. We shall not consider the issue of mode locking, which is out of the scope of this work.

Figure 5 summarizes the overall 2D scan results in the $\left(q_{95}, \Delta \Phi\right)$ space, for the same figures of merit as reported in Fig. 3, but assuming the $n=1$ coil configuration. The amplitude of the $n=1$ coil current is assumed to be the same as for the $n=2$ coil configuration in this study. Since the linear plasma response is computed here, the absolute amplitude of the coil current is of less interest in this study. The re-scaled figures of merit, shown in the left panel of Fig. 5, again yield linear correlations between the optimal coil phasing and $q_{95}$. The differences, compared to the $\mathrm{n}=2$ configuration, are the slope of the straight lines and the phase shift factor $\phi_{n}$ as defined in expression (1). For the $n=1$ configuration, the best fitting yields $a_{n}=0.018$ for all three figures of merit, and $\phi_{n}=50^{\circ}$ for the vacuum field criterion (plot a) and $\phi_{n}=110^{\circ}$ for the plasma response based two figures of merit (plots c and e). Noticeably, the phase difference for the optimal coil phasing, between 
the vacuum criterion and the plasma response criteria, is again $60^{\circ}$, the same as for the $n=2$ configuration.
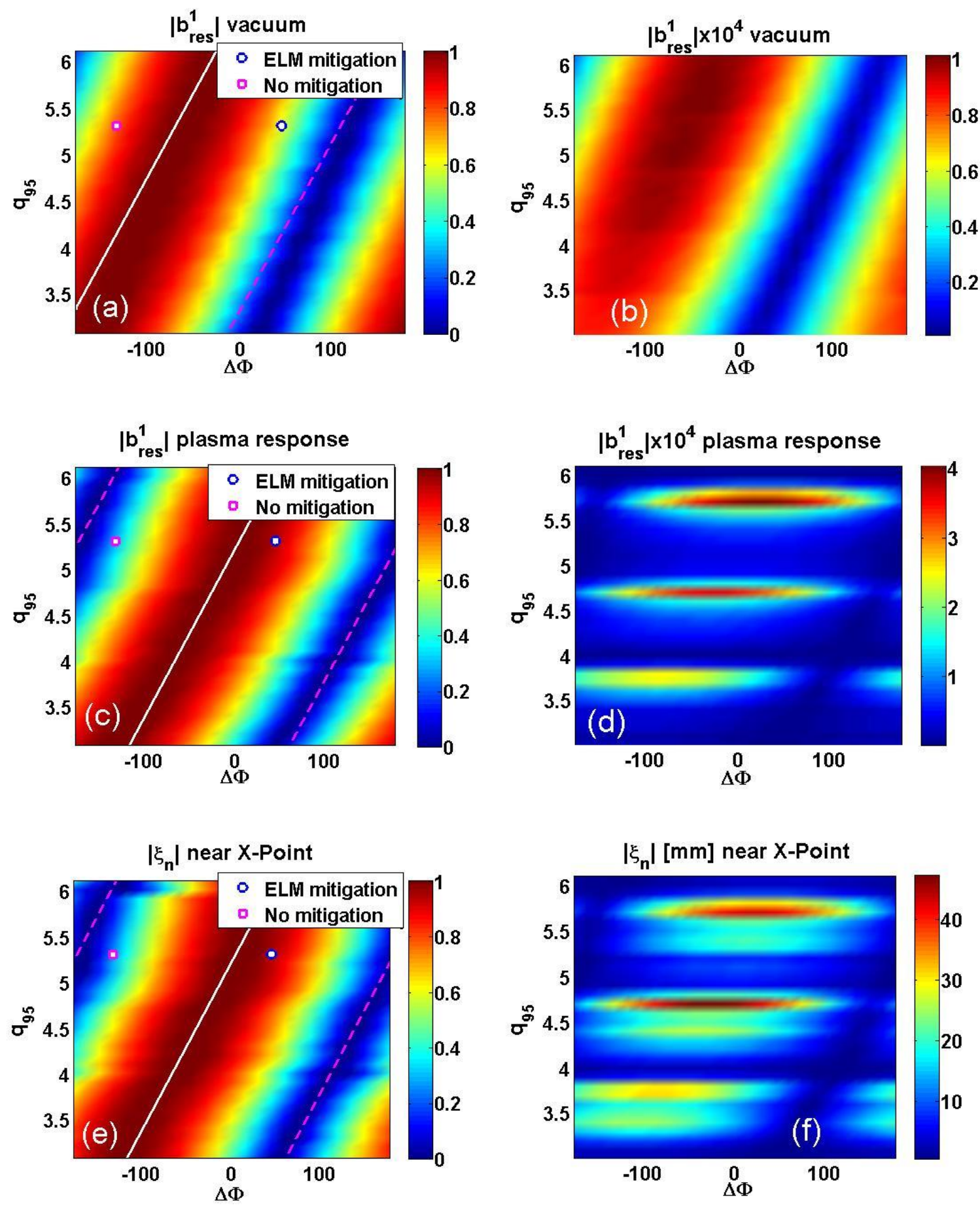

Figure 5. Various figures of merit computed in $2 \mathrm{D}$ parameter space $\left(\Delta \Phi, q_{95}\right)$, for (a-b) the amplitude of the outermost pitch resonant vacuum radial field component; (c-d) the amplitude of the outermost pitch resonant radial field component including the plasma response; and (e-f) the amplitude of the plasma surface displacement near the X-point. These quantities are all re-scaled to the range of [0, 1], at each given $q_{95}$, in the 
left panel plots (a, c, e), where the solid lines indicate the location of the peak value (of 1), and the dashed lines indicate the location of the minimum (of 0 ). The $n=1$ coil configuration is considered.

Two experimental data points, from shot 29160, where the coil phasing was varied during the same shot, are also plotted in Fig. 5 (left panel). Again the best alignment between the computed optimum for the coil phasing, and that from experiments, is obtained by taking into account the plasma response. The vacuum field criterion has difficulty in distinguishing between the ELM-mitigation phasing and the non-mitigation phasing.

Without re-scaling of the figures of merit to the range of $[0,1]$, the computed plasma response, presented by Fig. 5(d) and (f), again shows the multiple resonance effect as for the $n=2$ case. The difference is that smaller number of peaks are obtained with the $n=1$ coil configuration, within the same $q_{95}$ range of $[3.1,6.1]$.

Similar to Fig. 4, Fig. 6 compares the peak amplitude of the plasma response based figures of merit with the MARS-F computed stability of the $n=1$ ideal external kink (peeling) mode. The number of peaks in this case agrees with the number of stable windows along the $n q_{a}$ axis. However, again the peak response does not always occur at the same location of the stable windows. We explain this by several possible factors. First, in a full toroidal equilibrium with strong plasma shaping, the plasma response may not be due to a single stable eigenmode. On the contrary, the plasma response is generally a linear combination of the response of all stable eigenmodes in the plasma, with the same toroidal mode number $n$. If the dominant contributions to the plasma response can come from more than one eigenmode, we may expect more complex behavior in the relation between the response peaking and the stability of the ideal kink-peeling mode. We mention that strong experimental evidence in DIII-D has recently been reported, indicating the occurrence of the multi-mode response phenomenon [29]. 

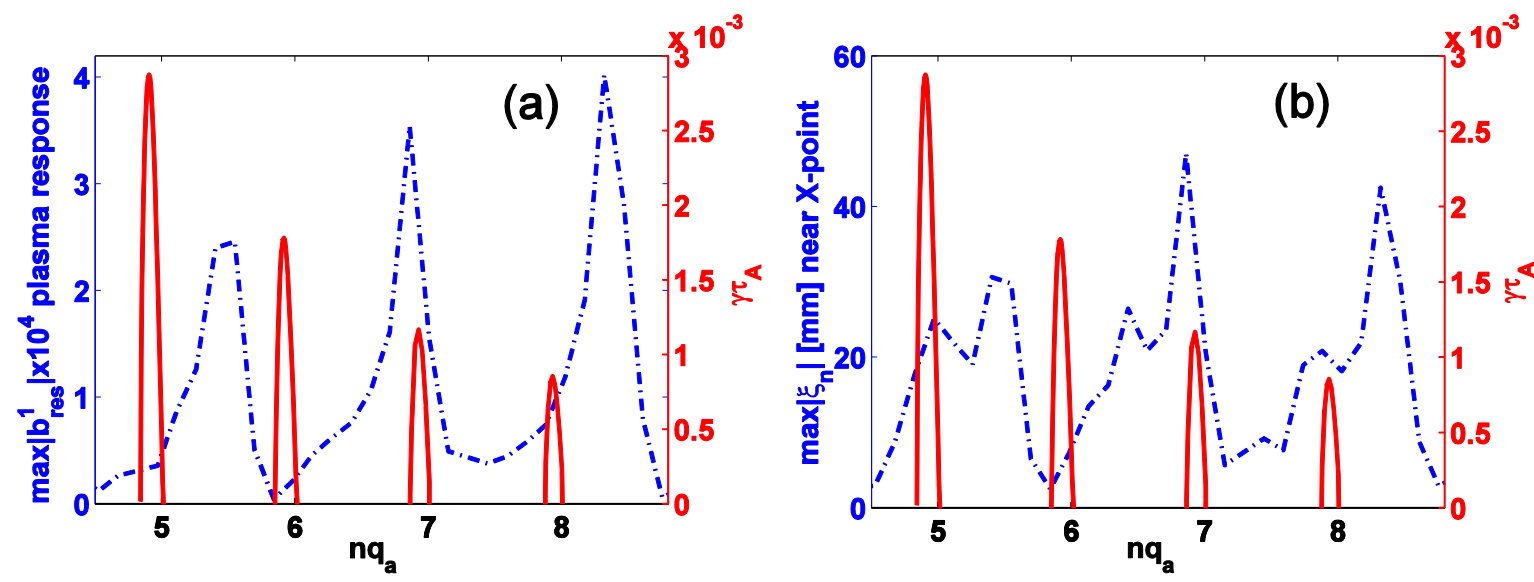

Figure 6. Growth rates (solid curves with right vertical axis in both plots, normalized by the on-axis toroidal Alfvén time) of the computed $n=1$ edge localized ideal peeling instability versus the edge safety factor $q_{a}$, plotted together with (a) the maximal amplitude (over all coil phasing angles $\Delta \Phi$ at fixed safety factor) of the last pitch resonant radial magnetic field component including the plasma response shown by the dash-dotted curve, and (b) the maximal amplitude of the X-point displacement shown by the dash-dotted curve, for the $n=1$ ELM control coil configuration.
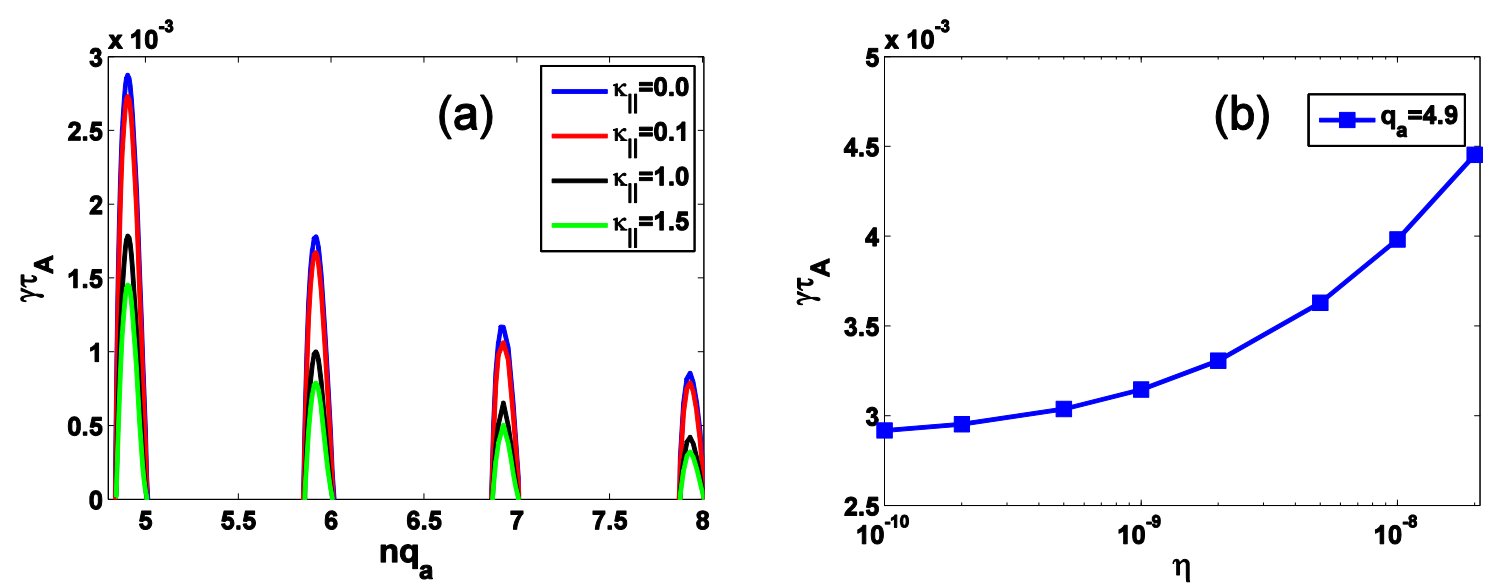

Figure 7. Effects of (a) the parallel sound wave damping, and (b) the plasma resistivity, on the stability of the $n=1$ edge localized peeling mode. Here $\kappa_{\square}$ is the numerical coefficient determining the strength of the parallel sound wave damping, $q_{a}$ the edge safety factor, $\eta=1 / S$ is the normalized core plasma resistivity, with $S$ being the Lundquist number.

Secondly, other physics effects beyond the ideal MHD model may affect the plasma response. In our response computations using MARS-F, these additional physics include the plasma toroidal flow, the plasma resistivity, as well as the parallel sound wave damping mimicking the ion Landau damping physics in a rotating plasma [8]. We have thus separately investigated all the aforementioned additional physics on the stability of the ideal kink- 
peeling mode for this ASDEX Upgrade equilibrium. We find that the plasma flow only introduces a Doppler shift to the mode frequency, without affecting the mode stability. This is similar to the findings from Ref. [27]. These eigenvalue computations are performed without including the effects from conducting structures. The (stabilizing) effect from the conducting structures (mainly the passive conducting plates in ASDEX Upgrade) should be weak for these peeling modes, which are strongly localized near the plasma edge, and the field perturbations decay fast in the vacuum region outside the plasma boundary.

The parallel sound wave damping, on the other hand, substantially reduce the mode growth rate, as shown by Fig. 7(a). This, in turn, should modify the plasma response in the stable window for the ideal kink-peeling mode. The plasma resistivity has a weak destabilizing effect on the peeling mode growth rate, shown by Fig. 7(b) as an example (at $q_{a}=4.9$ ). More direct investigation of the stable mode spectrum, in a full toroidal geometry with these additional physics, is computationally challenging (partially due to the presence of continuum spectra as well). Other approaches, such as Nyquist technique [30], may provide better insights into the plasma response in the stable domain. This is beyond the scope of the present work.

Another peculiar observation, shown in Fig. 5(b), is the fact that the computed vacuum resonant field near the plasma edge does not monotonically decrease with increasing $q_{95}$. This behavior is different from that of the $n=2$ coil configuration shown in Fig. 3(b). A detailed investigation, reported in Fig. 8, reveals that the amplitude of the resonant vacuum radial fields decays with different rates into the plasma region. Higher $q_{95}$ leads to faster decay of the field amplitude, as expected. As a result, further from the plasma boundary, the vacuum field does become smaller at higher $q_{95}$. However, near the plasma edge, this may not be the case, essentially due to the proximity effect to the RMP coils. We point out that this 
proximity effect depends on the coil phasing as well as on the toroidal mode number $n$. The coil phasing, for the three examples of $q_{95}$ shown in Fig. 8, is chosen to follow the straight solid line from Fig. 5(b). The vacuum field generally monotonically decays with $q_{95}$, for higher $n$ numbers ( $n$ larger than 1 in our study).

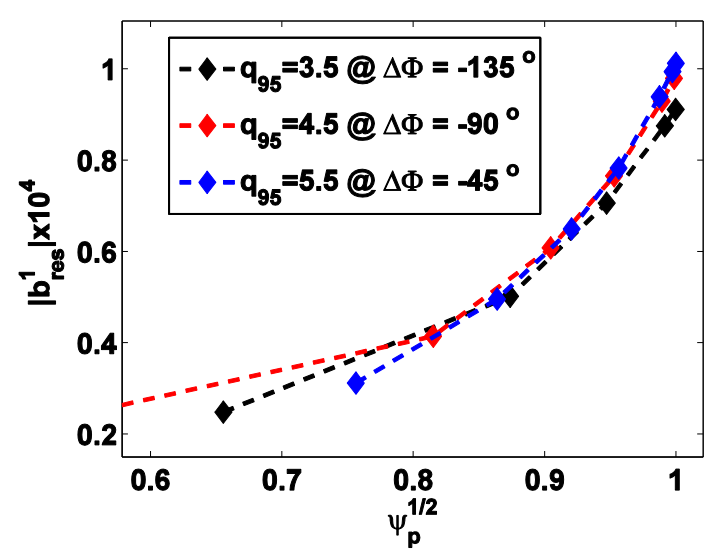

Figure 8. Comparison of the amplitude of the pitch resonant radial magnetic field components at the corresponding rational surface locations, of the $n=1$ vacuum RMP field, for three cases $\left(q_{95}=3.5\right.$, $\left.\Delta \Phi=-135^{\circ}\right),\left(q_{95}=4.5, \Delta \Phi=-90^{\circ}\right)$, and $\left(q_{95}=5.5, \Delta \Phi=-45^{\circ}\right)$, respectively. These three cases roughly align with the peak amplitude shown in Fig. 5(b).

\subsection{Plasma response to $n=3$ and 4 RMP fields}

We also performed similar systematic 2D scan of the plasma response for the $n=3$ and 4 RMP coil configurations, for the same series of the ASDEX Upgrade equilibria as for the $n=1$ and 2 fields, with results summarized in Figs. 9 and 10, respectively. As discussed before, the $n=3$ configuration is not the natural choice for the ELM control with the present set of $2 \times 8$ coils in ASDEX Upgrade. Nevertheless it is useful to provide computational data, for potential future experiments with $n=3$. Such experiments can serve two purposes: (i) to compare with similar experiments on other devices with the $n=3$ coil configurations, such as DIII-D and MAST; (ii) to study the effect of RMP fields with mixed toroidal spectrum on the ELM control in ASDEX Upgrade. 

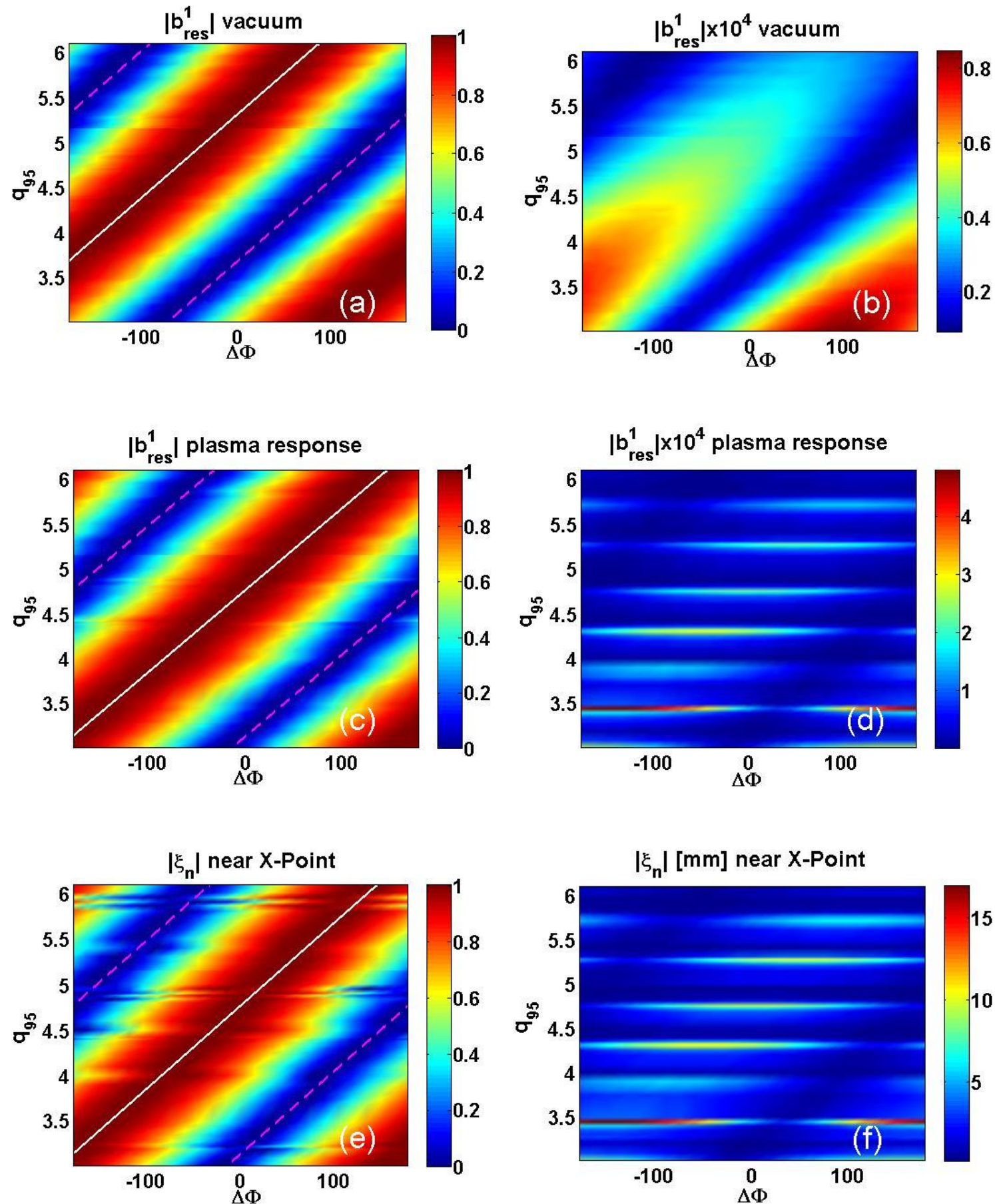

Figure 9. Various figures of merit computed in the 2D parameter space ( $\left.\Delta \Phi, q_{95}\right)$, for (a-b) the amplitude of the outermost pitch resonant vacuum radial field component; (c-d) the amplitude of the outermost pitch resonant radial field component including the plasma response; and (e-f) the amplitude of the plasma surface displacement near the X-point. These quantities are all re-scaled to the range of [0,1], at each given $q_{95}$, in the left panel plots (a, c, e), where the solid lines indicate the location of the peak value (of 1), and the dashed lines indicate the location of the minimum (of 0 ). The $n=3$ coil configuration is considered. 

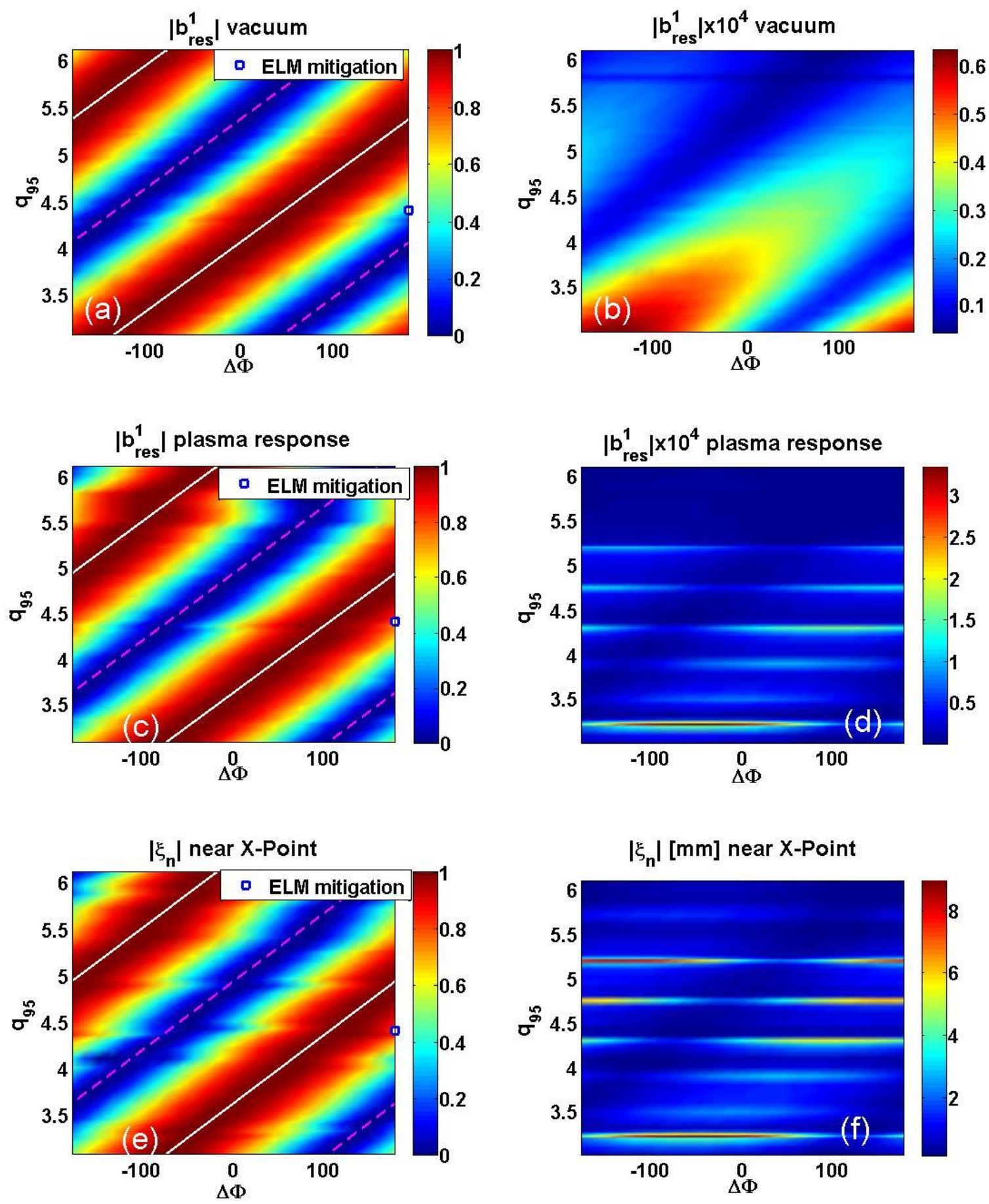

Figure 10. Various figures of merit computed in the 2D parameter space $\left(\Delta \Phi, q_{95}\right)$, for (a-b) the amplitude of the outermost pitch resonant vacuum radial field component; (c-d) the amplitude of the outermost pitch resonant radial field component including the plasma response; and (e-f) the amplitude of the plasma surface displacement near the X-point. These quantities are all re-scaled to the range of [0,1], at each given $q_{95}$, in the left panel plots (a, c, e), where the solid lines indicate the location of the peak value (of 1), and the dashed lines indicate the location of the minimum (of 0). One data point, where the ELM mitigation has been achieved in experiments, is shown by a filled circle. The $n=4$ coil configuration is considered, for which the experimental ELM control database is so far very limited. 
Both the $n=3$ and 4 computational results again show linear scaling of the optimal coil phasing with $q_{95}$. The slope coefficients, as well as the phase shift factor, as defined in Eq. (1), are now summarized in Table 1, for $\mathrm{n}=1-4$. Note that Table 1 reports the phase shift factor $\phi_{n}$ for three figures of merit separately, whilst the slope coefficient $a_{n}$ is the same. Furthermore, MARS-F linear response computations consistently show the same phase shift factor $\phi_{n}$, based on the plasma response induced amplitude of either the outmost pitch resonant radial field component or the X-point displacement, for each of the given $n$ coil configurations. This phase factor in turn is $60^{\circ}$ larger than that predicted by the vacuum radial field based figure of merit, for all $n=1-4$. This also confirms earlier studies performed for individual cases [17].

Table 1. Coefficients of linear fitting Eq. (1) for the MARS-F computed optimal coil phasing, for different choices of the toroidal mode number $\mathrm{n}$ for the ELM control coil configuration in ASDEX Upgrade plasmas.

\begin{tabular}{|c|c|c|c|c|}
\hline$n$ & $a_{n}$ & $\phi_{n}\left(b_{\text {vacuum }}^{1}\right)$ & $\phi_{n}\left(b_{\text {plasma }}^{1}\right)$ & $\phi_{n}\left(\xi_{n}\right.$ X-point $)$ \\
\hline 1 & 0.018 & $50^{\circ}$ & $110^{\circ}$ & $110^{\circ}$ \\
\hline 2 & 0.026 & $-50^{\circ}$ & $10^{\circ}$ & $10^{\circ}$ \\
\hline 3 & 0.027 & $-70^{\circ}$ & $-10^{\circ}$ & $-10^{\circ}$ \\
\hline 4 & 0.029 & $-100^{\circ}$ & $-40^{\circ}$ & $-40^{\circ}$ \\
\hline
\end{tabular}

Again, another observation from Figs. 9-10 is the presence of the multi-resonance effect of the plasma response, for both $n=3$ and $n=4$ coil configurations. Finally, the limited number of data points (from discharge 32094), where successful ELM mitigation were achieved with the $n=4$ coil configuration in ASDEX Upgrade, again align reasonably well with the region of large plasma response, as shown in Fig. 10(c, e), but does not align with the vacuum field based figure of merit. So far experiments are constrained by the choice of $q_{95}$ and the coil phasing (either even or odd) for the $n=4$ configuration. On the other hand, with 
the given constraint on the coil phasing for $n=4$, Fig. 10 provides possible $q_{95}$ values for achieving the best ELM mitigation, which can be validated in future experiments.

The first figure of merit that we have been using in this study, is associated with the pitch resonant radial field component at the outmost rational surface close to the plasma surface. This is, however, not the unique choice. We have also defined this figure of merit as the averaged value of all the resonant radial field components in the range of $[0.95,1]$ over the minor radius $s=\psi_{p}^{2 / 1}$. This essentially covers the whole edge pedestal region. The computed 2D scan results are very similar to that shown in Figs. 3, 5, 9, 10, for $n=1,2,3,4$, respectively. In fact all the numbers reported in Table 1 are exactly the same following the new definition, except that the phase shift factor for the vacuum field, $\phi_{n}\left(b_{\text {vacuum }}^{1}\right)$, is reduced by $15^{\circ}$ for the $n=1-3$ coil configurations. Note that $15^{\circ}$ is also the step size in our phase scan for $\Delta \Phi$. These results show that the scaling for the optimal coil phasing, that we find in this study, is relatively robust against the choice of the figures of merit.

\section{Effect of plasma shaping on the choice of optimal coil phasing}

So far no systematic theory efforts have been made to understand the effect of the plasma shaping on the plasma response. In the following, we shall gradually modify the shape of the plasma boundary in the upper outboard corner, starting from an equilibrium reconstructed for the ASDEX Upgrade discharge 30835 at $3200 \mathrm{~ms}$. We shall only consider the $n=2$ case, which is so far the most frequently adopted coil configuration in ASDEX Upgrade ELM control experiments.

Our primary concern is the effect of the upper triangularity of the plasma shape on the plasma response. To facilitate a systematic scan, we introduce an analytic model for modifying the existing plasma shape. Figure 11(a) shows a sketch of the model in the $(R, Z)$ 
plane. The black line represents the original plasma shape $L$, and the red line is a new shape $L^{\prime}$. To modify the plasma shape (mainly the upper triangularity) along the poloidal circumference between points $\mathrm{A}$ and $\mathrm{B}$, we multiply the $(R, Z)$ coordinates of the old shape $L$ by a factor involving Gaussian function. The new shape $L^{\prime}$ is represented by $\left(R^{\prime}, Z^{\prime}\right)$

$$
\begin{aligned}
& R^{\prime}=R\left(1+\hat{\delta} \exp \left(-\frac{\left(\phi-\phi_{m}\right)^{4}}{2 \kappa^{2}}\right)\right) \\
& Z^{\prime}=Z\left(1+\hat{\delta} \exp \left(-\frac{\left(\phi-\phi_{m}\right)^{4}}{2 \kappa^{2}}\right)\right)
\end{aligned}
$$

where $\hat{\delta}, \kappa$ and $\phi_{m}$ are the model parameters. The first parameter, $\hat{\delta}$, mainly controls the upper triangularity of the new shape, and shall further-on be referred to as the "triangularity parameter". The remaining two parameters are chosen to ensure sufficiently smooth connection of the new shape with the old shape at points A and B.

In this work, we shall fix $\kappa=0.5$ and $\phi_{m}=0.5323$, and only adjust $\hat{\delta}$ to obtain a family of plasma shapes, shown in figure 11(b), where the black solid line again represents the original plasma shape from discharge 30835 at $3200 \mathrm{~ms}$. The new shapes have either smaller $(\hat{\delta}<0)$ or larger $(\hat{\delta}>0)$ triangularity, compared to the original shape. The red squares in Fig. 11(b) indicate the location of the RMP coils.

We point out that this procedure results in very minor modification to the safety factor profile. In fact with the same total plasma current and the same toroidal equilibrium field, and for the new plasma boundary shapes shown in Fig. 11(b), all the self-consistently computed safety factor profiles nearly overlap, with only a small variation of the edge safety factor value as plotted in Fig. 11(c). This is important in isolating the physics effects associated with the plasma shaping in further studies. 

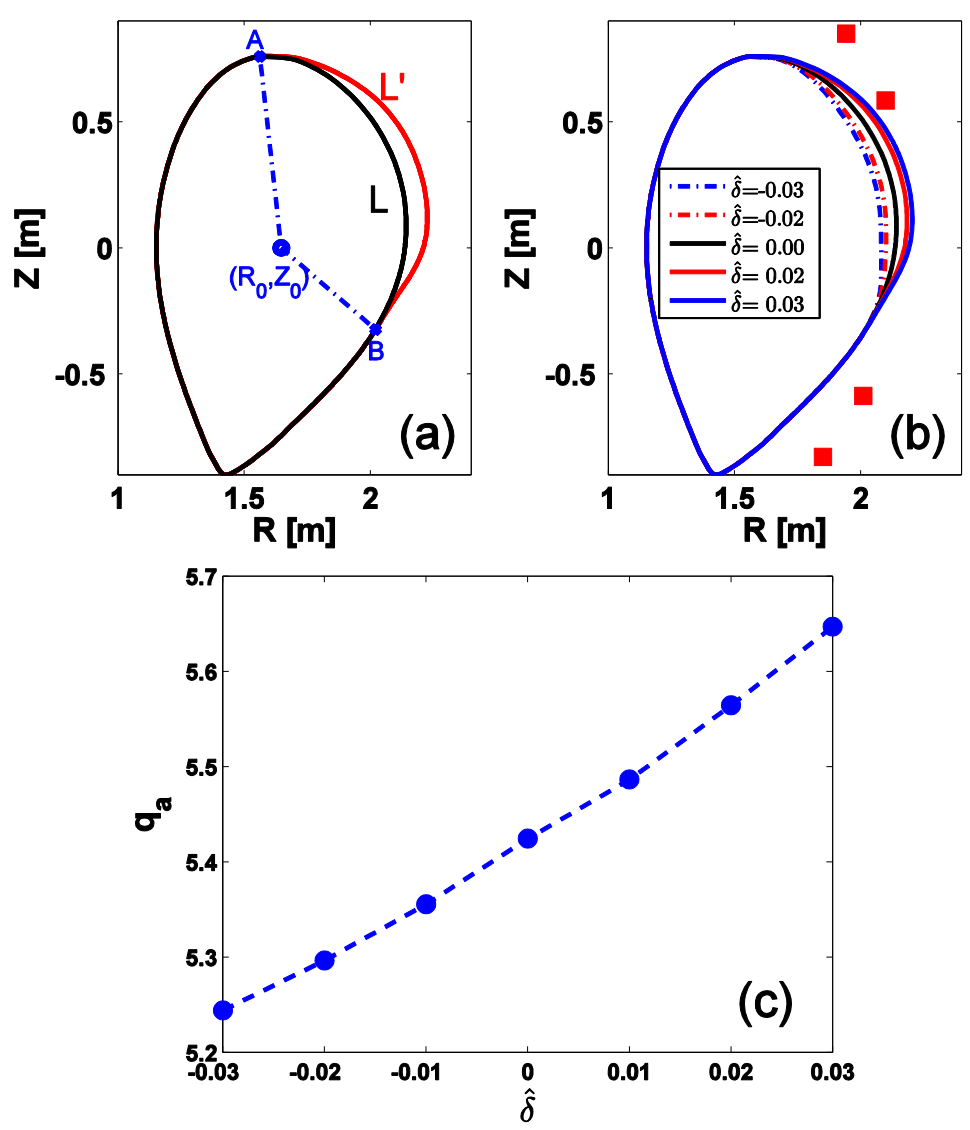

Figure 11. Modification of the upper low field side quarter of the plasma boundary shape, based on the ASDEX Upgrade discharge 30835, with (a) showing a sketch of the modification, (b) a family of the plasma boundary shapes together with the RMP coil locations, and (c) the slight variation of edge safety factor $q_{a}$, associated with the change of the shaping. The shaping parameter $\hat{\delta}=0$ corresponds to the original shape for 30835, which is also denoted by the black curve in (b).

We again perform the plasma response computations by scanning the coil phasing angle $\Delta \Phi$, for each of the new equilibria with varying plasma shape following our model. The same three figures of merit are used, in order to measure the plasma response. The corresponding results are plotted in Fig. 12 (a-c), respectively, for various choices of the triangularity parameter $\hat{\delta}$. We shall first discuss the optimal coil phasing that maximizes the corresponding figures of merit. These optimal coil phasing angles, as functions of $\hat{\delta}$, are summarized in Fig. 12(d), where the black curve with stars and the red curve with squares represent the optimal phasing based on the vacuum and the total response field, respectively. 
The blue curve with squares shows $\Delta \Phi$ that maximizes the amplitude of the plasma surface displacement near the $\mathrm{X}$ point.
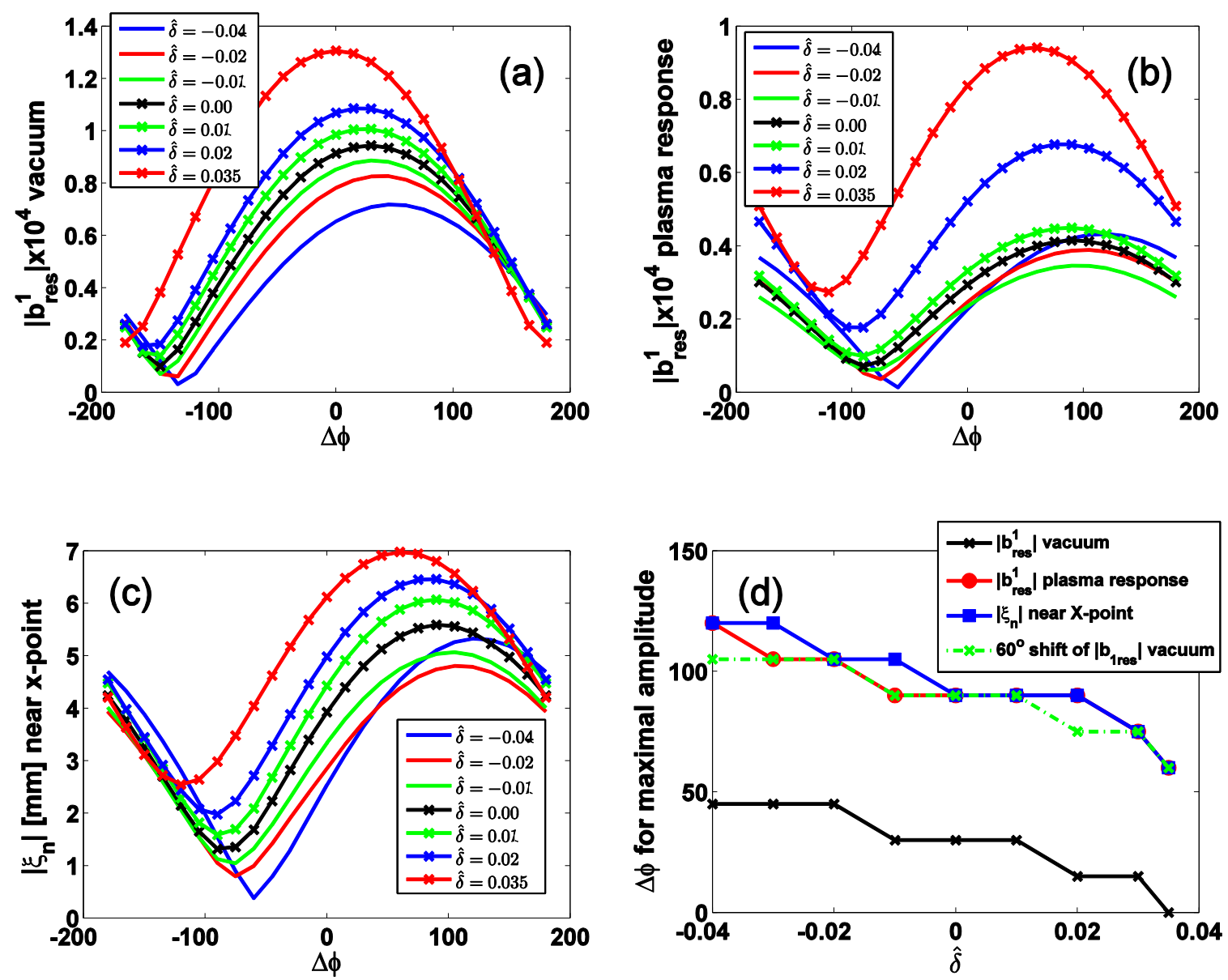

Figure 12. Effects of gradual variation of the plasma shaping (with the shaping parameter $\hat{\delta}$ ), on the plasma response, with (a) and (b) showing the amplitude of the last pitch resonant radial field component of the vacuum field and the total field including the plasma response, respectively, (c) showing the amplitude of the plasma surface displacement near the X-point, and (d) the optimal coil phasing $\Delta \Phi$ that maximizes various figures of merit. The green dashed line in (d) is obtained by adding a positive 60 degrees shift to the black solid line showing the case for the vacuum field.

A couple of interesting points can be made here. First, the optimal coil phasing varies with the plasma shape. For our case, the optimal phasing generally decreases with the triangularity parameter $\hat{\delta}$. This is predicted by all three figures or merit including even the vacuum based criterion. The difference is that the latter again yields optimal phasing that is roughly $60^{\circ}$ less than that predicted by the two plasma response based criteria. In fact, by 
shifting the vacuum criterion based curve up by $60^{\circ}$ (dash-dotted green curve), we obtain reasonably good overlap of all three top curves shown in Fig. 12(d).
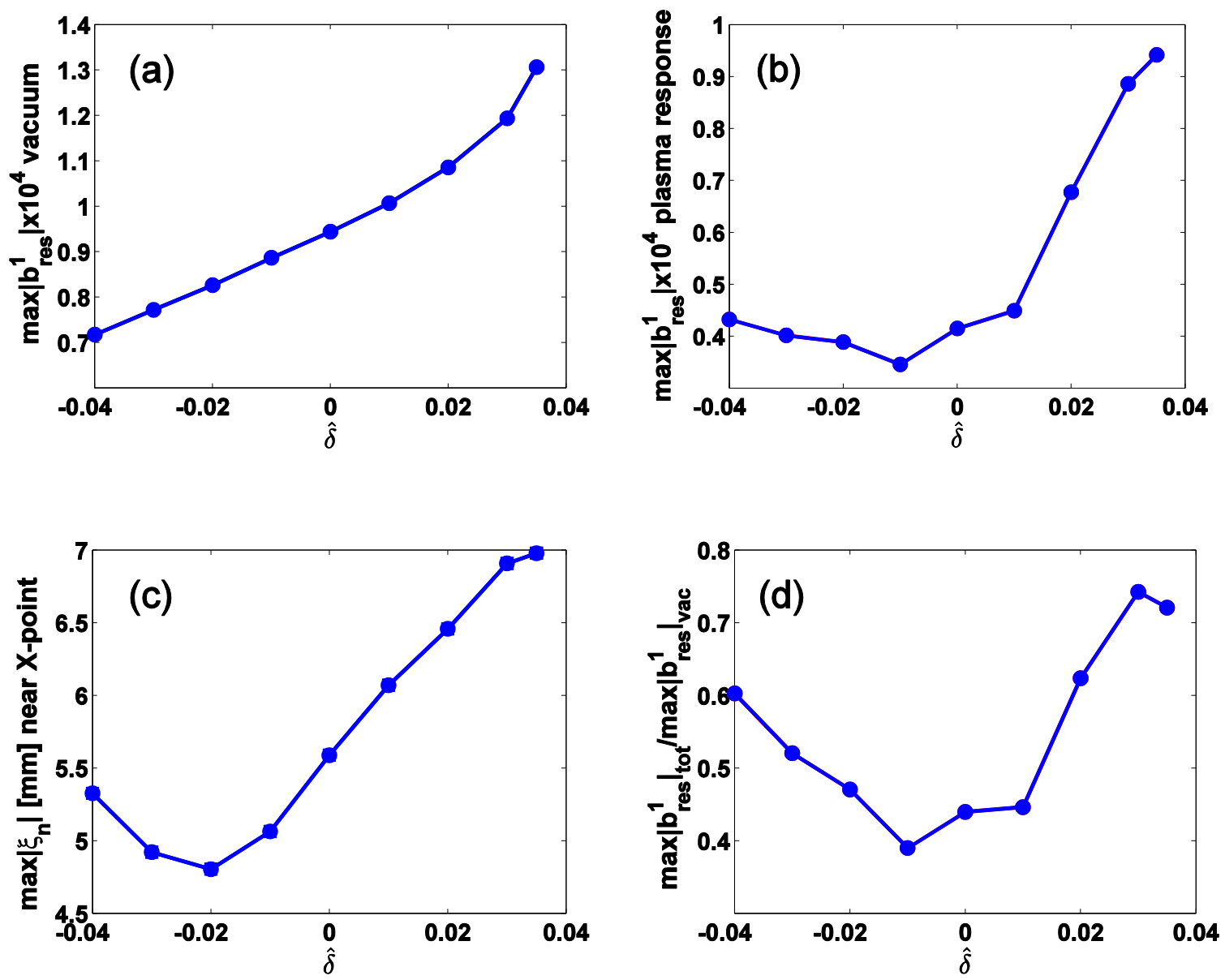

Figure 13. Effects of the plasma boundary shaping (with the shaping parameter $\hat{\delta}$ ) on the best achievable figures of merit (over all possible choices of the coil phasing), for (a) and (b) the maximal amplitude of the last pitch resonant radial field component of the vacuum field and the total field including the plasma response, respectively, (c) the maximal amplitude of the plasma surface displacement near the X-point, and (d) the ratio of the maximum resonant field amplitude at the last rational surface, between the vacuum field and the total response field.

Secondly, it is evident that the variation of the optimal phasing with $\hat{\delta}$ cannot be simply explained by the proximity of the plasma surface to the upper row of coils. The fact that the vacuum field and the total response field yield a similar tendency for the optimal coil phasing (except for the $60^{\circ}$ shift), indicates that even the plasma response is not playing a 
dominant role here. Since the safety factor profiles are also similar for these cases, as has been pointed out before, the only major factor left, that can explain the computed variation of the optimal phasing with the plasma shaping, is the modification of the poloidal spectrum of the vacuum field, due to the change of the plasma shape. This is eventually related to the change of the poloidal angle.

On the other hand, the modification of the upper triangularity does significantly change the distance between the plasma surface and the upper row of coils in our case, as already shown by Fig. 11(b). This proximity effect leads to significant variation of the computed amplitude of the vacuum and the plasma response fields, as evident from Fig. 12(a-c). This is more clearly shown in Fig. 13(a-c), where the peak amplitude (over all the coil phasing angles) of the three figures of merit are plotted, respectively, against $\hat{\delta}$. It is interesting to note though, that whilst the vacuum field amplitude monotonically increases with $\hat{\delta}$ (plot (a)), indicating predominantly the coil proximity effect, the plasma response introduces secondary effect that leads to non-monotonic dependences (plots (b-c)). By taking the ratio of the total response field amplitude to that of the vacuum field, as shown in Fig. 13(d), we cancel the coil proximity effect. The resulting curve shows the effect of the plasma response to the variation of the upper trangularity of the plasma shape. This effect is non-monotonic, and the minimal response occurs at $\hat{\delta}$ near -0.01 , i.e. with a plasma shape that is slightly less triangular than the original 30835 shape.

\section{Conclusion and discussion}

The MARS-F code, based on the single fluid resistive plasma model in the presence of toroidal flow, has been used to compute the plasma response to the applied RMP fields in the ASDEX Upgrade ELM control experiments. Two important factors affecting the ELM control, 
namely the edge safety factor and the plasma triangularity, are systematically investigated. The optimal toroidal phase difference (coil phasing), of the coil currents between the upper and lower sets of coils in ASDEX Upgrade, is the key element of investigation.

In our modelling, the optimal coil phasing is defined as that maximizing either the amplitude of the resonant poloidal harmonic of the perturbed radial magnetic field (effectively the width of the magnetic island) near the plasma edge, or the radial displacement of the plasma surface near the X-point. Toroidal computations show that, when the plasma response is taken into account, these two figures of merit always yield the same optimum for the coil phasing. Moreover, across the reasonably large database for the $n=2$ coil configuration, these optima seem to be in good correlation with the best ELM mitigation found in experiments.

Modelling shows a roughly linear scaling of the optimal coil phasing with $q_{95}$, for various choices of the toroidal number mode $n(n=1,2,3,4)$ for the ELM control coils. The coefficients of the linear scaling are different for different toroidal mode numbers, and are numerically determined in this work (Table 1). Such scaling, which is found to be relatively insensitive to the definition of the figures of merit, can be useful for guiding the choice of coil phasing in future experiments in ASDEX Upgrade.

Computations also reveal multiple resonance effect for the amplitude of plasma response, in terms of both figures of merits defined in this study, while continuously varying the edge safety factor. The plasma response experiences strong amplification in narrow windows of $q_{95}$, as has previously been reported [18]. We find that the peak amplitude of the plasma response is related to, but not always exactly correlated with, the marginal stability of the ideal edge peeling mode. Other physics, such as the multi-mode response, the plasma resistivity, the toroidal flow, as well as the parallel sound wave damping, may also play roles. 
Modelling shows that the optimal coil phasing also varies with the upper triangularity of the plasma shape. Three factors are identified here, which affect the plasma response with varying shaping: the proximity of the plasma boundary to the upper row of RMP coils, the change of the poloidal spectrum of the applied vacuum field near the plasma edge, and the difference in the plasma response due to the change of the plasma shape. For the $n=2$ coil configuration at similar $q_{95}$ (corresponding to the low- $q$ discharge 30835), the optimal coil phasing monotonically decreases with increasing the upper triangularity. This is mainly due to the variation of poloidal spectrum of the applied vacuum field. The amplitude of the peak vacuum field monotonically increases with the upper triangularity, primarily due to the coil proximity effect. On the other hand, the amplitude of the plasma response does not follow a monotonic variation with the plasma shaping. This is essentially due to the plasma response effect.

\section{Acknowledgement}

We thank Dr. H. R. Koslowski for helpful suggestions improving the manuscript. Dr. H. Meyer's suggestion for the sensitivity check of the figures of merit is also gratefully acknowledged. This work has been carried out within the framework of the EUROfusion Consortium and has received funding from the Euratom research and training programme 2014-2018 under grant agreement No 633053 and from the RCUK Energy Programme [grant number EP/I501045]. Work is also part funded by National Natural Science Foundation of China (NSFC) [grant numbers 11405029, 11275047, 11505021 and 11428512] and by National Magnetic Confinement Fusion Science Program under grant No. 2015GB104004. The views and opinions expressed herein do not necessarily reflect those of the European Commission. 


\section{References}

[1] Loarte A et al 2007 Nucl. Fusion 47 S203

[2] Evans T et al 2004 Phys. Rev. Lett. 92235003

[3] Hawryluk R J et al 2009 Nucl. Fusion 49065012

[4] Liang Y et al 2007 Phys. Rev. Lett. 98265004

[5] Kirk A et al 2010 Nucl. Fusion 50034008

[6] Suttrop W et al 2011 Phys. Rev. Lett. 106225004

[7] Jeon Y M et al 2012 Phys. Rev. Lett. 109035004

[8] Liu Y Q et al 2010 Phys. Plasmas 17122502

[9] Heyn M F et al 2008 Nucl. Fusion 48024005

[10] Becoulet M et al 2009 Nucl. Fusion 49085011

[11] Liu Y Q et al 2011 Nuc.l Fusion 51083002

[12] Ferraro N M 2012 Phys. Plasmas 19056105

[13] Chapman I T et al 2012 Plasma Phys. Control. Fusion 54105013

[14] Chapman I T et al 2014 Nucl. Fusion 55013004

[15] Orain F et al 2013 Phys. Plasmas 20102510

[16] Park G et al 2010 Phys. Plasmas 17102503

[17] Ryan D A et al 2015 Plasma Phys. Control. Fusion 57095008

[18] Haskey S R et al 2015 Plasma Phys. Control. Fusion 56035005

[19] Liu Y Q et al 2015 Nucl. Fusion 55063027

[20] Kirk A et al 2015 Nuc.l Fusion 55043011

[21] Liu Y Q et al 2000 Phys. Plasmas 73681

[22] Turnbull A et al 2013 Phys. Plasmas 20056114

[23] Lanctot M et al 2011 Phys. Plasmas 18056121 
[24] Liu Y Q et al 2012 Plasma Phys. Control. Fusion 54124013

[25] Kirk A et al 2011 Plasma Phys. Control. Fusion 53065011

[26] Liang Y et al 2011 Nuc.l Fusion 51073001

[27] Liu Y Q et al 2010 Plasma Phys. Control. Fusion 52045011

[28] Suttrop W et al 2014 25th IAEA Int. Conf. on Fusion Energy, St Petersburg, Russia, EX/P1-23

[29] Paz-Soldan C et al 2015 Phys. Rev. Lett. 114105001

[30] Liu Y Q et al 2000 Phys. Rev. Lett. 84907 